\title{
EDITORIAL
}

\section{Zika Virus Infection: Are We Aware?}

Zika virus (ZIKV) is an enveloped, single stranded RNA virus which is transmitted by daytime-active Aedes mosquitoes. Its name comes from the Zika Forest of Uganda, where the virus was first isolated in 1947. In human, the virus causes a mild illness known as Zika fever, Zika, or Zika disease, which since the 1950s has been known to occur within a narrow equatorial belt from Africa to Asia.

In April 2007, the first outbreak outside of Africa and Asia occurred on the island of Yap in the Federated States of Micronesia, which was initially thought to be dengue, chikungunya or Ross River disease. ${ }^{1}$ In 2014, the virus spread eastward across the Pacific Ocean to French Polynesia, then to Easter Island and in 2015 to Mexico, Central America, the Caribbean, and South America, where the Zika outbreak has reached pandemic levels. ${ }^{2}$ In February 2016, the WHO declared the virus outbreak a public health emergency of international concern. A number of countries have issued travel warnings.

The virus is mainly spread by the Aedes aegypti mosquito, which is commonly found throughout the tropical and subtropical Americas, but also by Aedes albopictus, "Asian tiger" mosquitoes that now have become widespread up to the Great Lakes area of the United States. ${ }^{3}$ Studies show that the extrinsic incubation period in mosquitoes is about 10 days. ${ }^{4} \mathrm{Zika}$ virus can migrate between humans through sexual contact and it can also cross the placenta, affecting an unborn fetus. A mother already infected with Zika virus near the time of delivery can pass on the virus to her newborn around the time of birth, but this is rare. The vertebrate hosts of the virus are primarily monkeys and humans.

The potential societal risk of Zika virus can be delimited by the distribution of the mosquito species that transmit it (its vectors). The global distribution of the most cited carrier of Zika virus, A. aegypti, is expanding due to global trade and travel. ${ }^{5}$
Zika virus is related to dengue, yellow fever, Japanese encephalitis, and West Nile viruses. Common symptoms of infection with the virus include mild headaches, maculopapular rash, fever, malaise, conjunctivitis, and joint pains. Within two days, the rash started fading, and within three days, the fever resolved and only the rash remained. Thus far, Zika fever has been a relatively mild disease of limited scope, with only one in five persons developing symptoms, with no fatalities and poses a significant threat only in pregnancy.

It is difficult to diagnose Zika virus infection based on clinical signs and symptoms alone due to overlaps with other arboviruses that are endemic to similar areas. ${ }^{6}$ The methods currently available to test for Zika antibodies cross-react with dengue antibodies. Zika IgM ELISA test should be considered indicative of a recent flavivirus infection. Plaque reduction neutralization tests (PRNT) can be performed to measure virus specific neutralizing antibodies to zika virus and may be specific. ${ }^{7}$ Zika virus can be identified by RT-PCR in acutely ill patients. However, there are no commercially available diagnostic tests for zika virus.

Symptoms can be treated with rest, fluids, and paracetamol while non-steroidal anti-inflammatory drugs should be used only when dengue has been ruled out .

Zika virus infections have been confirmed in several infants with microcephaly; Guillain- Barré syndrome and other neurologic conditions may be complications of Zika virus. ${ }^{8}$

Work has begun towards developing a vaccine for Zika virus. It may take two years to develop a vaccine, but 10 to 12 years may be needed before an effective Zika virus vaccine is approved by regulators for public use. ${ }^{9}$

The good news is, the risk for zika virus infection in Bangladesh is low as the countries affected are far away. But the bad news is, a swarm of aedes mosquitoes are waiting to greet zika virus if it ever reaches our country. It is the high time to design a National strategy and policy to identify and prevent zika virus disease in Bangladesh and ensure emergency preparedness plan. 
We have to act in a coordinate way to control the mosquitoes, inform people about the risks, keep a check on cases, step up research to understand the disease and develop vaccines.

(J Banagladesh Coll Phys Surg 2016; 34: 1-2)

\section{Prof. Md. Enamul Karim}

Professor and Head, Department of Medicine

Dhaka Medical College.

\section{Reference:}

1. Altman, L.K. (3 July 2007). "Little-Known Virus Challenges a Far-Flung Health System”. New York Times.

2. McKenna, Maryn (13 January 2016). “Zika Virus: A New Threat and a New Kind of Pandemic”.Germination. Retrieved 18 January 2016.

3. Moritz UG Kraemer et al. (2015) The global distribution of the arbovirus vectors Aedes aegypti and Ae. albopictus. eLife 2015;4:e08347. http://dx.doi.org/10.7554/eLife. 08347

4. Hayes, E. B. (2009). “Zika Virus Outside Africa”. Emerging Infectious Diseases 15(9):1347-50. doi:10.3201/ eid1509.090442.PMC 2819875. PMID 19788800.
5. Kraemer, Moritz UG; Sinka, Marianne E.; Duda, Kirsten A.; Mylne, Adrian QN; Shearer, Freya M.; Barker, Christopher M.; Moore, Chester G.; Carvalho, Roberta G.; Coelho, Giovanini E. (7 July 2015).”The global distribution of the arbovirus vectors Aedes aegypti and Ae.albopictus”.ELife 4: e08347. doi:10.7554/ eLife.08347.PMC 4493616. PMID 26126267.5

6. Fauci, Anthony S.; Morens, David M. (13 January 2016). "Zika Virus in the Americas - Yet Another Arbovirus Threat". New England Journal of Medicine: 160113142101009. doi:10.1056/NEJMp1600297. PMID 26761185.

7. CDC (2016) Updated diagnostic testing for Zika, chikungunya, and dengue viruses in US Public Health Laboratories. Memorandum of 13 January 2016. CDC, Division of Vector-Borne Diseases, Arboviral Diseases and Dengue Branches. http://www.cdc.gov/zika/pdfs/ denvchikvzikv-testing-algorithm.pdf

8. "WHO sees Zika outbreak spreading through the Americas”. Reuters. 25 January 2016. Retrieved 25 January 2016.

9. James Cook (27 January 2016). “Zika virus: US scientists say vaccine '10 years away'-BBC News”. BBC News. Retrieved 28 January 2016. 